\title{
A minicomputer method for generating dichotic word pairs*
}

\author{
JAMES L. KNIGHT, JR. and BARRY H. KANTOWITZ \\ Purdue University, West Lafayette, Indiana 47906
}

A computer method is described for generating dichotic word pairs. The Purdue Laboratory system is implemented with a DEC PDP-12 computer.

Studies of auditory selective attention and auditory laterality often employ dichotically presented word pairs as stimuli. In dichotic presentation, two different words are presented simultaneousiy, one word to each ear. Generally it is of considerable importance to ensure that word pairs are (a) accurately synchronized with respect to onset time (Treisman \& Riley, 1969; Moray \& O'Brien, 1967) and (b) are of precisely controlled duration. The present paper describes a method employed at the Purdue Human Information Processing Laboratory to generate dichotic word pairs which rigorously meet these criteria. Onset alignment and duration are accurate to better than 10 microsec.

Although numerous methods have been previously employed for generation of dichotic word pairs, these methods usually have one or more undesirable deficiencies. Simple and inexpensive methods generally require multiple recordings on a stereo tape recorder. Words intended for one ear are recorded on one track in time with a metronome. A second recording pass is then made to record words intended for the other ear. Left and right tracks may then be fed to a dual-channel scope for visual verification of onset synchrony and word duration. However, only poor stimulus control may be achieved using such methods. More sophisticated approaches have achieved better stimulus control via highly specialized equipment such as tape recorders with eight or more movable heads (e.g., SPACELOOP, Beetle \& Chapman, 1968). The general unavailability and expense of such specialized equipment make such methods of limited use in most psychological laboratories. A more recent and flexible approach (e.g. Yntema \& Trask, 1963: Treisman \& Riley. 1969) uses computers to store digitized representations of two or more words in core memory. Selected word pairs are put out via digital-to-analog converter (DAC) channels under program control. While this approach allows generation of precisely synchronized word pairs. large computers with extensive memory storage capacity $(>32 \mathrm{~K})$ have been required. Also, control of word durations is not always accomplished. Besides the expense of such large computers, it is often not desirable or feasible to

\footnotetext{
*This system was developed under the auspices of Office of Education Grant OEG-5-72-0025 (509). The authors gratefully acknowledge the assistance of Dr. Terry Yates throughout the development of this system.
}

dedicate such a machine to on-line generation and presentation of stimuli.

With the advent of the general-purpose, relatively inexpensive laboratory minicomputer, a new approach to the generation of dichotic speech appeared open. However, lack of memory storage capacity was a serious impediment to the use of minicomputers for this purpose. To store digitized representations of two .5 -sec words plus an operating program simultaneously requires approximately $16 \mathrm{~K}$ storage locations, while the majority of laboratory minicomputers are equipped with $8 \mathrm{~K}$ or less of core storage. The system described in the present paper circumvents this difficulty while maintaining good speech quality. Furthermore, processing of individual words to uniform duration is both fast $(<60 \mathrm{sec})$ and easy. A final feature of this system is that dichotic word pairs may be presented on-line during an experiment directly under computer control. Because this eliminates the need to prerecord stimulus tapes of dichotic word pairs, it is possible for $E$ to vary the presentation order of dichotic pairs on the basis of S's responses.

\section{HARDWARE REQUTREMENTS}

The primary hardware requirement is a minicomputer with at least $8 \mathrm{~K}$ central memory. In addition, a real-time computer-controlled clock, three analog-to-digital converter (ADC) channels, two independent DAC channels. and some form of high-speed mass data storage are needed. Optional equipment includes a single-channel triggered scope, stereo tape recorder, three tunable low-pass audio filters, and a voltage amplitude peak clipper. At the Purdue Laboratory, this system has been implemented with a Digital Equipment Corporation (DEC) PDP-12 equipped with $8 \mathrm{~K}$ central memory and standard DEC peripherals. LINCtape serves as the mass storage medium. The computer may, of course, be other than a DEC machine. However, at present the operating software is available only in DEC PAL III language. The hardware setup (including optional equipment) is schematized in Fig. 1. Operation of the hardware may be best understood with reference to the following overall system description.

\section{DESCRIPTION OF THE OPERATING SYSTEM}

A controlling program DICHOTIC ${ }^{1}$ written in PAL III controls the hardware and produces dichotic word pairs in a two-stage process. In Stage 1. monaural words are digitized, adjusted to proper duration. and permanently stored on the mass storage medium. In Stage 2. processed words are retrieved in pairs from mass storage and are presented on-line to $S$ s or are recorded on audio tape for later presentation. 


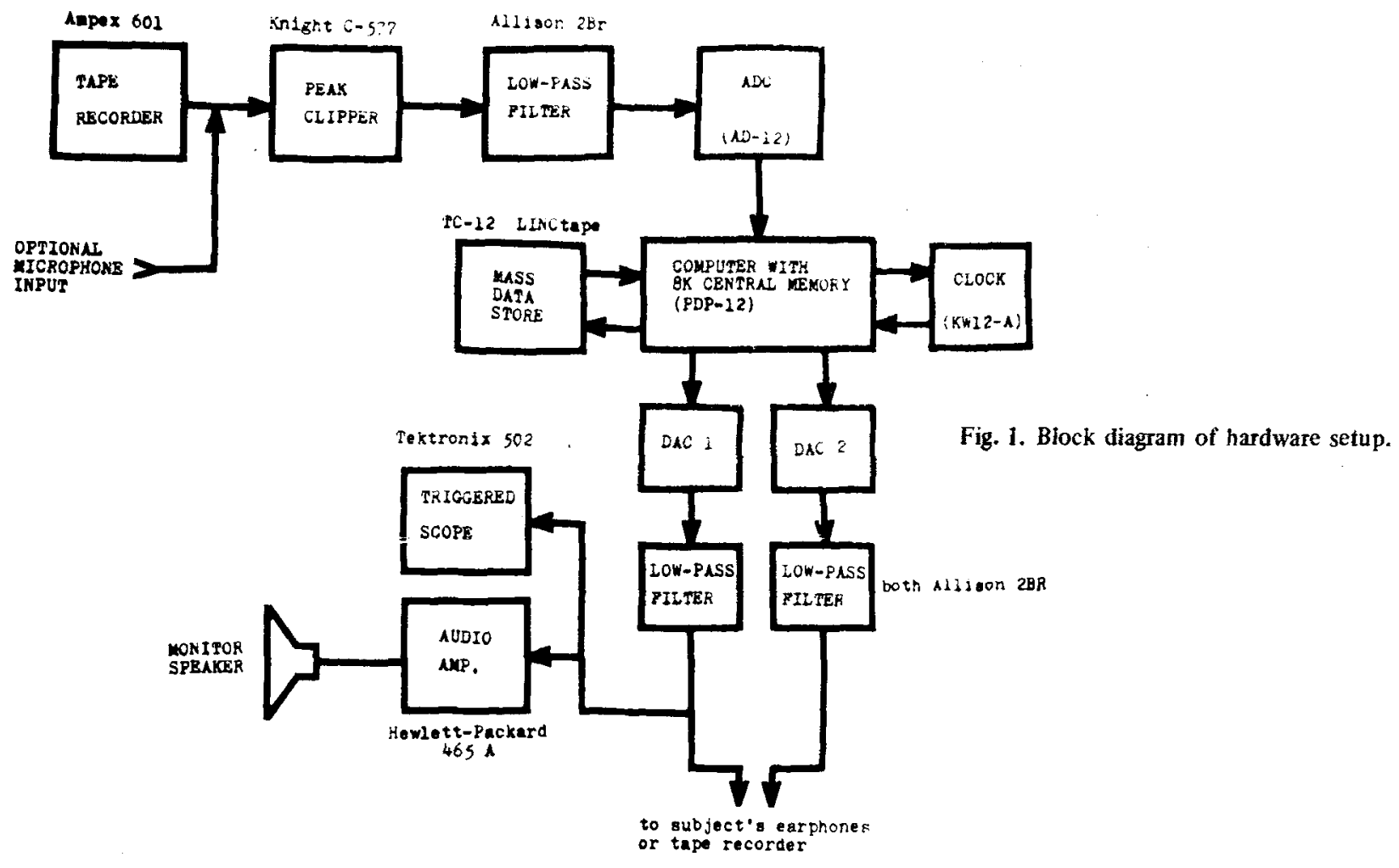

Stage 1

A single monaural word is initially presented to the computer. While this may be directly from a microphone (see Fig. 1), it is usually desirable to prerecord a monaural list of all words to be used as stimuli. During this initial recording, care should be taken to ensure that recording level is constant. A satisfactory method is to employ a well-practiced speaker and to monitor recording level on a high-quality VU meter. This precaution ensures that final dichotic word pairs will be of uniform intensity. The speech signal undergoes some preliminary filtering and peak clipping as shown in Fig. 1. The bit deletion algorithm (to be described shortly) employed by DICHOTIC requires signal amplitude changes to be as large as possible and to cover the entire ADC range $( \pm 1 \mathrm{~V})$. To accomplish this, a high-amplitude signal is fed through a peak clipper which limits signal voltage to $\pm 1 \mathrm{~V}$ to protect the ADC. This clipped signal then enters a low-pass filter tuned to attenuate frequencies above the Nyquist frequency $\left(\mathrm{f}_{\mathrm{N}}=\right.$ $1 / 2$ sampling frequency) to reduce distortion.

When initially started, DICHOTIC remains in a wait loop continually checking the ADC output in anticipation of word arrival. DICHOTIC uses a simple threshold $( \pm 15 \mathrm{mV})$ reference to determine that a word is arriving at the ADC. When word arrival is sensed, a real-time clock is enabled and the ADC switches to a fast sample mode. DICHOTIC begins to store a digitized representation of the word in an upper $4 \mathrm{~K}$ memory field, taking and storing one sample at each clock pulse. Thus, 4,096 data storage locations are available, while
DICHOTIC itself occupies about $1,400_{8}$ lower field locations.

At this point, the primary limitation of minicomputers in speech processing-lack of sufficient core to permit storage of a lengthy digitized word-is encountered. An obvious solution is to employ slow sampling rates. For speech signals, this is generally unsatisfactory. Words spoken in isolation are usually about $.5 \mathrm{sec}$ in duration. Since only 4,096 storage locations are available, sampling rates must be limited to about $8 \mathrm{~K} / \mathrm{sec}$. This limits the theoretically usable signal frequency spectrum to $0-4 \mathrm{kHz}$ (e.g., see McGillem \& Cooper, 1971, p. 5-170). Our experience at the Purdue Laboratory has indicated that this is insufficient for satisfactory intelligibility of many speech sounds, particularly $/ \mathrm{s} /, / \mathrm{f} /$, and $/ \theta /$.

As an alternative to slow sampling rates, DICHOTIC reduces information storage demands on the central processor by tracking the input waveform with larger ADC step sizes. This is accomplished by deleting the 4 lowest order bits of each 10-bit ADC sample. Thus, 6 significant bits (including a sign bit) remain from each sample. DICHOTIC packs two successive 6-bit samples in each 12-bit computer word to effectively double available core capacity. This allows sampling rates up to $16 \mathrm{~K} / \mathrm{sec}$. The technique of increasing step size, of course, adds distortion, but the signal degradation is considerably less than that which results from comparable reduction in sampling rate. This point will be considered later in more detail. DICHOTIC continues to sample, digitize, pack, and store the input signal until 
all available upper core storage is filled.

Once digitized, packed, and stored, the word is repeatedly decoded and played back by the computer through DAC 1 (see Fig. 1). This presentation triggers the scope horizontal sweep (set to $50 \mathrm{msec} / \mathrm{cm}$ ) and allows the operator to visually inspect the digitized word. An audio amplifier and speaker connected in parallel with the scope allow the operator to listen to the digitized word. The rate at which the computer repeats the word is synchronized with the scope sweep rate so that the word appears as a stationary pattern on the scope. At the end of each playback, DICHOTIC generates an "end-of-word marker" pulse on DAC 1 which appears as a pip on the display scope. The user adjusts the location of this marker by varying the voltage $( \pm 1 \mathrm{~V})$ applied to a second $A D C$ channel. In the Purdue PDP.12 implementation, the internal ADC Channel 5 serves this function. The end-of-word marker is aligned with the actual word end as seen on the scope. This setting is not critical, the only requirement being that a nonsignal gap is not left between the actual word end and the end-of-word marker. At each playback, DICHOTIC terminates the word at the point specified by the end-of-word marker, thus allowing the operator to evaluate subjectively the end-of-word setting.

When satisfied with the end-of-word adjustment, the operator signals this fact to DICHOTIC by setting a switch. The portion of the digitized word preceding the current end-of-word marker is stored in a temporary working area of the mass storage medium. A subroutine PROCRUSTES now adjusts word duration to the desired value. The range of possible durations depends upon the actual sampling rate chosen by the user. At high sampling rates $(14-16 \mathrm{~K} / \mathrm{sec})$, approximate word duration range is $200-600 \mathrm{msec}$. PROCRUSTES examines the operator-specified end-of-word setting to determine if the partially processed word is too long or too short. If the word is too long, PROCRUSTES deletes a middle portion of the word and then juxtaposes the remaining portions to fill the gap thus created. If the word is too short, PROCRUSTES expands the word by repeating a middle portion of the word. The initial locus of this deletion or repetition is adjustable via a third $A D C$ channel (ADC Channel 6 is used on the PDP-12 version). After PROCRUSTES completes its adjustments, the processed word is presented visually and auditorily. The partially processed word is repeatedly retrieved from the temporary working area, adjusted by PROCRUSTES, and displayed until the operator is satisfied with the result. The processed word is then preserved in a specified location of the mass storage medium for retrieval during Stage 2. Thus, a file of processed uniform-duration words is built up during Stage 1. Stage 1 processing usually requires less than $1 \mathrm{~min}$ of operator time per word.

Stage 2

During Stage 2, processed words are retrieved from storage and presented as dichotic pairs over the two DAC channels. By initially setting a sense switch, the operator specifies that Stage 2 rather than Stage 1 operation of DICHOTIC is desired. The operator now specifies the two words which are to comprise the dichotic pair. Any two words processed during Stage 1 may be accessed. Upper and lower $4 \mathrm{~K}$ memory fields each hold the digitized representation of one word. Because the lower 4K field also holds DICHOTIC and any additional experiment-control software, DICHOTIC first transfers all lower core information to the temporary working area of the mass storage medium. Remaining in lower core is a rudimentary routine to restore lower core information from the temporary working area. Also remaining is a routine for decoding and presenting the digitized words on the two DAC channels. This latter routine is highly efficient, since very little time is available for this operation. In synchrony with the real-time clock, successive samples of each word are alternatively decoded from their half-word representation and recorded on audio tape (off-line) or sent directly (on-line) to S's earphones via DACs 1 and 2. At output, the words are sent through low-pass filters which attenuate distortion above the Nyquist frequency. Because output alternates between the two words at each clock pulse, an unavoidable minimum output asynchrony of 8.6 microsec results; however, this is still several orders of magnitude better than the asynchrony inherent in the methodology of most published dichotic studies. Following dichotic word-pair presentation, lower core information is restored from the working area and another dichotic pair is presen ed. For on-line operation, the interpair interval is determined by how rapidly words may be retrieved from the mass storage medium. With LINCtape this may be relatively long-20 to $30 \mathrm{sec}$. When implemented with disk storage facility, DICHOTIC can generate sequences of dichotic pairs with interpair intervals of $70 \mathrm{msec}$-faster than 2 pairs per sec.

\section{THE BIT DELETION ALGORITHM}

Initially, the bit deletion algorithm was favored over slow sampling rate as a method of reducing information storage load. Most minicomputers utilize bipolar 8- or 10-bit (including sign bit) ADCs. Since DEC machines utilize 12-bit computer words, either 2 or 4 bits are unused at each sample. Thus, it was clear that available storage capacity would be wasted if the slow sampling technique were chosen. Also, a 10-bit sample was probably unnecessarily precise, since this represented a step size of less than $2 \mathrm{mV}$ over a range of $\pm 1 \mathrm{~V}$. This step size was not much larger than the ambient circuit noise level. Finally, temporal information regarding speech signals may be more crucial in maintaining intelligibility than is amplitude information. Some evidence for this is the moderately high intelligibility of infinitely peak-clipped speech. Increasing sample step size (i.e., deleting low order bits) and sampling rate 
increases the accuracy of temporal information in the digitized representation at the expense of less important amplitude information.

Both bit deletion and slow sampling rate techniques were tried in DICHOTIC. and the superiority of the bit deletion algorithm was qualitatively apparent. To provide a more quantitative comparison of the two methods, a FOCAL program, SIGSIM, ${ }^{1}$ was written to generate simulated signals under a variety of conditions. Signal frequency, sampling rate, and number of bits per sample were parameters used by SIGSIM. Simulated signals were submitted to Fourier analysis using a DEC Fast Fourier Transform (FFTD) program. This analysis confirmed the superiority of the bit deletion algorithm. For example, sampling a $3-\mathrm{kHz}$ sine wave at $16 \mathrm{~K}$ samples/sec with 6-bit accuracy yielded $9.7 \%$ more energy at $3 \mathrm{kHz}$ than did $8 \mathrm{~K}$ samples/sec with 10-bit sampling. In addition, the frequency spread between half-energy points was approximately twice as great for the slow sampling rate as for the fast rate. While the bit deletion algorithm is superior to sampling rate reduction in the current application, the advantage will not remain for all numbers of bits per sample and sampling rates. With the sampling rates employed here, 4 - or 5; bit samples represent the minimum amplitude accuracy necessary for adequate intelligibility. The bit deletion algorithm should be far more advantageous with 16-bit word minicomputers such as the Data General NOVA or the Hewlett-Packard HP series, since two 8-bit samples can be packed together. A more detailed study of the relationship between sample amplitude accuracy and temporal accuracy is currently underway.

\section{REFERENCES}

Beetle, D. H., Jr., \& Chapman, W. D. Flexible analog time compression of short utterances. IEEE Transactions on Audio \& Electroacoustics, 1968, AU-16, 1, 12-20.

McGillem, C. D., \& Cooper, G. R. Continuous and discreet signal and system analysis. New York: Holt, Rinehart \& Winston, 1971.

Moray, N., \& O'Brien, T. Signal-detection theory applied to selective listening. Journal of the Acoustical Society of America, 1967, 42, 765-772.

Treisman, A. M., \& Riley, J. G. A. Is selective attention selective perception or selective response? A further test. Journal of Experimental Psychology, 1969, 79, 27-34.

Yntema, D. B., \& Trask, F. P. Recall as a search process. Journal of Verbal Learning \& Verbal Behavior, 1963, 2, 67-74.

\section{NOTE}

1. A copy of the operating program DICHOTIC is available upon request to the first author. Documentation on the FOCAL program SIGSIM is also available. 